\title{
Técnica de minilaparotomía "Minilap" en pacientes sometidos a prostatectomía radical por cáncer de próstata
}

\author{
Gómez Veiga F, Barbagelata López A, Ponce Díaz-Reixa J, Fernández Rosado E, \\ González Martín M.
}

Servicio de Urología Complexo Hospitalario Universitario Juan Canalejo. A Coruña.

Actas Urol Esp. 2007;31(6):593-602

\section{RESUMEN}

TECNICA DE MINILAPAROTOMIA “MINILAP” EN PACIENTES SOMETIDOS A PROSTATECTOMIA RADICAL POR CANCER DE PRÓSTATA

Introducción: El cáncer de próstata es en la actualidad el tumor mas frecuente en el varón. Muchos de estos tumores con la utilización del PSA y diagnóstico precoz se detectan en fases localizadas. La prostatectomía radical (PR.) sigue siendo el método de tratamiento más usado, si bien dicha cirugía no está exenta de riesgos

Los refinamientos técnicos y en la técnica como las incisiones mínimas minilaparotomía (Minilap), han contribuido a mejorar los resultados. Presentamos nuestra experiencia con la técnica de Minilap en pacientes sometidos a PR..

Material y métodos: En el período comprendido entre abril de 1997 y septiembre de 2005 realizamos 110 PR. según técnica de Minilap. En todos los casos se utilizaron incisiones de 7-8 cms. Para la realización de esta técnica utilizamos un separador multivalvas desarrollado en el propio Hospital. La edad media era de 65 años (4779) por estadios, 39 (35,4\%) eran T1c, 64 (58,3\%) cT2 y 7 (6,3\%), cT3. En 68,2\% el Gleason era $\leq 6,34$ (30,9\%), 7 y $1(0,9 \%), 8$.

Resultados: El estadio patológico fue pT2 86(38,1\%), pT3 en 21 (19\%), pT4 1(0,9\%) y 2(1,8\%) pT0. En 9 (8\%) casos se presentaron complicaciones postoperatorias. Ninguno precisó reintervenciones y $101(90 \%)$ no presentaron complicaciones perioperatorias. La mediana de estancia postoperatoria fue de 4 dias y 97 pacientes $(88,8 \%)$ fueron dados de alta en los 5 primeros días. Al año un $92.3 \%$ presentan continencia urinaria completa o fugas minimas y un $40 \%$ vida sexual activa.

Conclusiones: Técnicas mínimamente invasivas para la cirugía de cáncer de próstata como la Minilap pueden realizarse de forma habitual con buenos resultados y sin necesidad de una curva de aprendizaje prolongada.

Palabras clave: Cáncer de próstata. Prostatectomía radical. Minilap.

\section{ABSTRACT}

RADICAL PROSTATECTOMY WITH MINILAPAROTOMY “MINILAP” FOR PATIENTS WITH PROSTATE CANCER

Introduction: prostate cancer is the most frequent tumor in males. The use of PSA contributes to diagnose tumors with low stage. Radical Prostatectomy (R.P.) is the gold standard to treat this tumor; however such is not exempt of risks. Different technical modifications like minimal incisions minilaparotomy "minilap" had contributed to improve results. We review our experience with Minilap on patients underwent a RP.

Material and Methods: Between April 1997 and September 2005 carry out 110 RP with Minilap technique. All cases were performed with minimal incision 7-8 cm of length. We use and specific retractor developed in our hospital. Median age at time of surgery was 65 (47-79). Clinical stage in 39 (35.4\%) were T1c, 64(58.3\%) T2 and $7(6.3 \%)$ T3. Sixty eight percent were Gleason score $\leq 6,34(30.9 \%) 7$ and $1(0.9 \%)$ Gleason 8.

Results: In 86 cases (38.5\%) pathological stage were pT2 , 21(19\%) pT3, 1(0.9\%) pT4 and 2 (1.8\%) pT0. Nine patients $(8 \%)$ had postoperative complications. No re-interventions were necessary and $101(90 \%)$ were discharge without per operatives complications. Mean length of stay was 4 days and 97(88.8\%) of patients were discharge with only five days length of stay. Urinary continence rate with a year like minimal follow up is $92.3 \%$ and $40 \%$ preserve sexual activity.

Conclusions: Minimal invasive techniques like minilap can be done in regular form with good results and without long learning curve.

Keywords: Prostate cancer. Radical prostatectomy. Minilap. 
$\mathrm{E}^{\mathrm{s}}$ cáncer de próstata es en la actualidad la neoplasia más frecuente en el varón en el mundo occidental, siendo en USA la predicción en el 2006 de más de 230.000 nuevos casos, con un número estimado de 27.300 muertes por dicha causa, convirtiéndola en la tercera causa de muerte ${ }^{1}$.En nuestro país en la década del 2000 y en particular en el 2002 según datos del Ministerio de Sanidad se diagnosticaron 13.500 nuevos $\operatorname{casos}^{2}$. La puesta en marcha de los programas de detección precoz y cribado sistemático han emergido gran número tumores potencialmente susceptibles de ser tratados con intención curativa.

En el contexto del tratamiento del cáncer localizado en los últimos años se han abierto múltiples alternativas, pero la cirugía radical y más concretamente la PR. retropúbica (PRR.) sigue siendo el estándar de tratamiento. Así aproximadamente un $45 \%$ de los pacientes son tratados con esta técnica ${ }^{3}$.

Si bien la técnica quirúrgica es un buen método para el manejo de esta neoplasia, la misma no está exenta de morbilidad, especialmente en lo referente a la disfunción eréctil, continencia e impacto en calidad de vida ${ }^{4}$. En cualquier caso el mejor conocimiento anatómico de la pelvis y en particular de la anatomía prostática junto con los cambios de técnicas nos permiten en la actualidad una mejoría en los resultados de esta cirugía, haciéndola menos agresiva con menos impacto en la calidad de vida del paciente. En esta línea y en base a las modificaciones técnicas se desarrollaron métodos menos invasivos como la Minilap que reduciendo las incisiones y apoyada en un buen soporte técnico de separadores específicos, nos permiten realizar esta cirugía facilitando la recuperación del paciente en su proceso postoperatorio.

En este trabajo revisamos nuestra experiencia con la técnica de Minilap descrita por Marshall ${ }^{5}$ para realizar la PR.

\section{MATERIAL Y MÉTODOS}

En el período comprendido entre abril de 1997 y septiembre de 2005 se realizaron 110 PR según técnica de Minilap. La edad media de los pacientes fue de 65 años con un rango de 47-79, la mediana de PSA fue $7,9 \mathrm{ng} / \mathrm{ml}$ con rango 2,8-180.
El paciente con PSA de 180 se trataba un enfermo con 3 biopsias previas negativas, y la cuarta con un solo cilindro de diez positivo y Gleason 6 . El Gleason medio de la biopsia fue 6 con un rango de 4-9. El rango de porcentaje de cilindros positivos osciló entre 10\% y 100\%, con mediana de $37 \%$. La mediana de volumen prostático fue de 45 c.c. y rango 13-125 (Tabla 1).

Para la clasificación de estadios utilizamos la TNM de 1997. Por estadíos, 39 (35,4\%) eran Tlc, $64(58,3 \%)$ eran cT2 y $7(6,3 \%)$ cT3. En $75(68,2 \%)$ el Gleason era $\leq 6$, en $34(30,9 \%) 7$ y $1(0,9 \%) 8$. (Tabla 1).

Tabla 1. Datos descriptivos de la serie

\begin{tabular}{|c|c|c|}
\hline \multicolumn{3}{|l|}{ Edad } \\
\hline Media & 65 & $(47-79)$ \\
\hline Mediana & 66 & \\
\hline \multicolumn{3}{|l|}{ PSA } \\
\hline Media & 12 & $(2.8-180)$ \\
\hline Mediana & 7.9 & \\
\hline \multicolumn{3}{|l|}{ Tacto rectal } \\
\hline Normal & 62 & $(56,4 \%)$ \\
\hline Anormal & 48 & $(43,6 \%)$ \\
\hline \multicolumn{3}{|l|}{ Estadio clínico } \\
\hline CT1 & 39 & $(35,4 \%)$ \\
\hline $\mathrm{CT} 2$ & 64 & $(58,3 \%)$ \\
\hline CT3 & 7 & $(6,3 \%)$ \\
\hline \multicolumn{3}{|l|}{ Gleason Biopsia } \\
\hline$\leq 6$ & 75 & $68,2 \%$ \\
\hline 7 & 34 & $30,9 \%$ \\
\hline $8-10$ & 1 & $0,9 \%$ \\
\hline \multicolumn{3}{|l|}{$\%$ Cilindros positivos } \\
\hline Media & 37 & $(10-100)$ \\
\hline Mediana & 30 & \\
\hline \multicolumn{3}{|l|}{ Volumen prostático } \\
\hline Media & 45 & $(13-125)$ \\
\hline Mediana & 42 & \\
\hline \multicolumn{3}{|l|}{ Estadio Patológico } \\
\hline T0 & 2 & $1,8 \%$ \\
\hline $\mathrm{T} 2 \mathrm{a}$ & 42 & $38,2 \%$ \\
\hline $\mathrm{T} 2 \mathrm{~b}$ & 44 & $40 \%$ \\
\hline T3а & 16 & $14,5 \%$ \\
\hline T3b & 5 & $4,5 \%$ \\
\hline $\mathrm{T} 4 \mathrm{a}$ & 1 & $0,9 \%$ \\
\hline \multicolumn{3}{|l|}{ Gleason PSA } \\
\hline$\leq 6$ & 39 & 35,5 \\
\hline 7 & 49 & 44,5 \\
\hline $8-10$ & 20 & 18,7 \\
\hline Márgenes positivos & 21 & $19 \%$ \\
\hline pT2 & 10 & $11,4 \%$ \\
\hline$\geq \mathrm{pT} 3$ & 11 & $50 \%$ \\
\hline
\end{tabular}


La técnica anestésica utilizada en todos los casos fue la endotraqueal general. Para la colocación del paciente se realizó con una flexión amplia del mismo en posición de Trendelenburg (Fig. 1), Con una incisión en línea media de 7-8 cm. (Fig. 2) justo a $1 \mathrm{~cm}$. por debajo de la sínfisis del pubis. Tras la apertura de piel, subcutáneo y disección muscular, se seccionó la aponeurosis de los rectos y se amplió en sus extremos 1-2 cm. para así facilitar la movilización de los tejidos abdominales y ganar espacio con los separado-

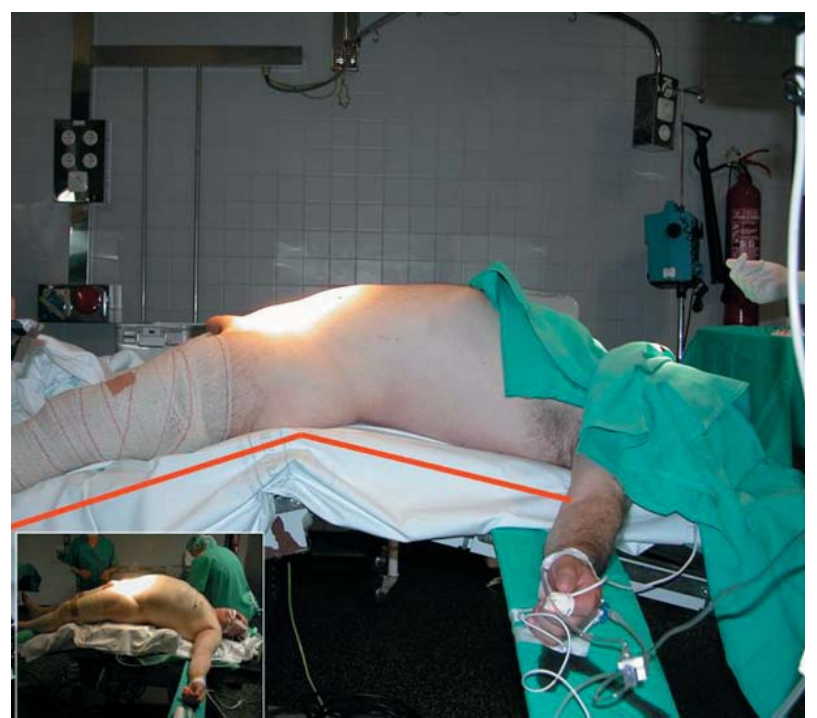

FIGURA 1. Posición de paciente. En recuadro paciente con elevado indice de masa corporal.

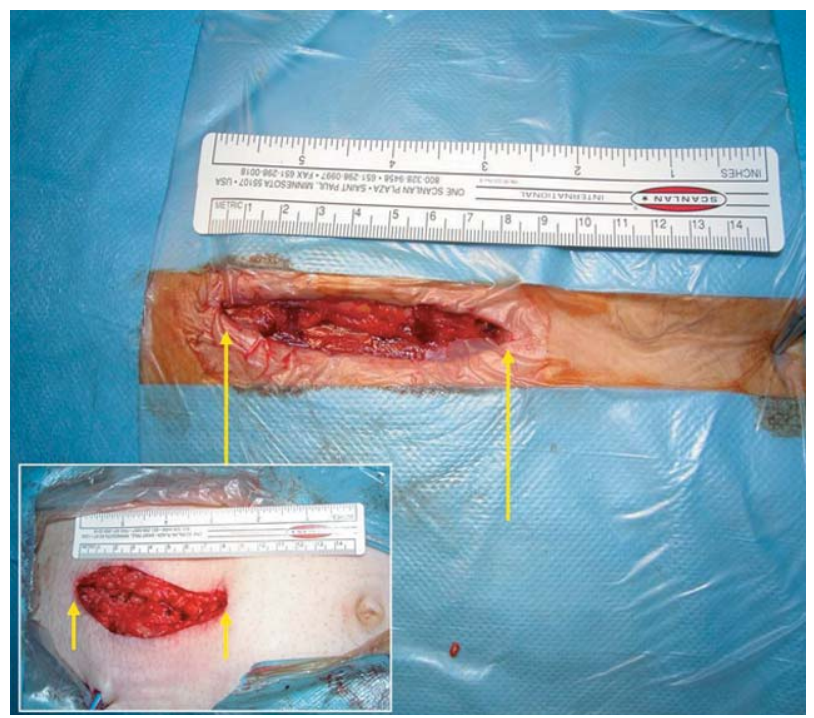

FIGURA 2. Incisión quirurgica. En recuadro incisión al final de la cirugia. res. Hecha esta maniobra, se procede a la colocación del separador desarrollado en el Servicio de Urología (Dr. M. González Martín) con valvas múltiples adaptables sobre soporte externo. La colocación de las valvas se planteó siempre en la misma disposición, dos en la porción abdominal inferior en ángulo, y otras dos superiores que desplazan el peritoneo y retraen la vejiga desarrollando un espacio circular que facilita el acceso al suelo pélvico (Fig. 3). En todos los casos se realizó linfadenectomía iliaco-obturatriz de estadiaje (Fig. 4). El motivo de la disección en casos de riesgo o no riesgo siguió criterios oncológicos y académicos para su ejecución.

En 6 pacientes $(5,4 \%)$, en la PR. no se hizo conservación de cuello vesical, en 16 (14,5\%) se conservó el cuello vesical y en 88 ((80\%) se realizó conservación de cuello y disección modificada de ápex con preservación de uretra

La técnica de la PR. incluyó la movilización de todo el conjunto prostato-seminal. La disección del complejo venoso se realizó con un punto de sutura 2/0 monofilamento, liberación e incisión de la fascia endopélvica siguiendo en la mayoría de los casos liberación amplia de uretra apical (Fig.5). El cuello se preservó con liberación del mismo hasta su inserción en próstata (Fig. 6).

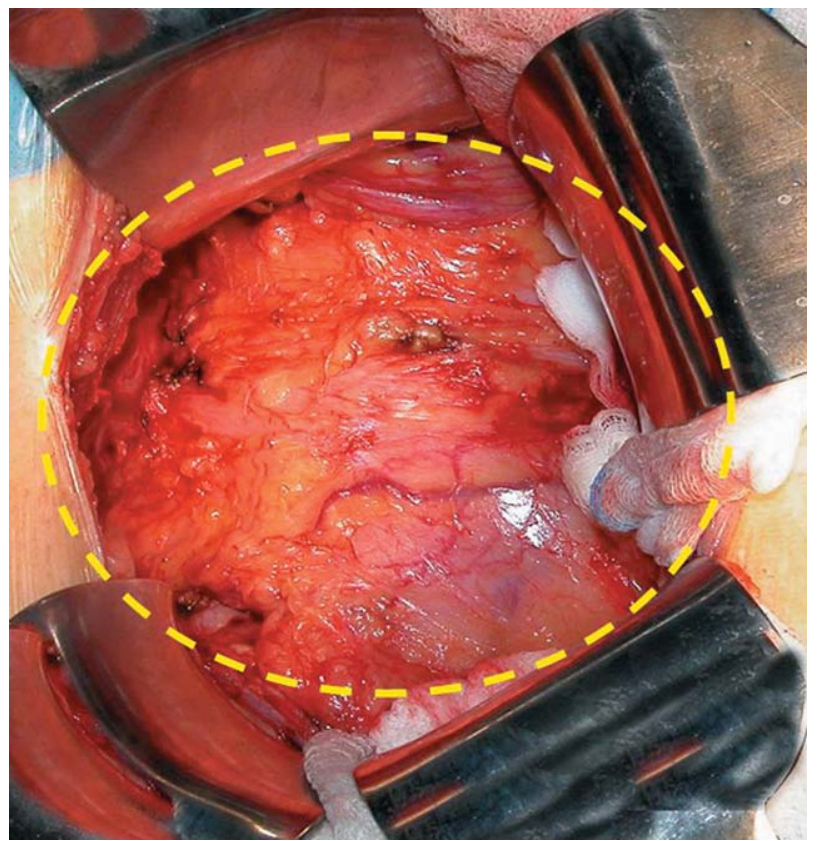

FIGURA 3. Separador multivalvo autoestático. Disposición del campo quirurgico. 


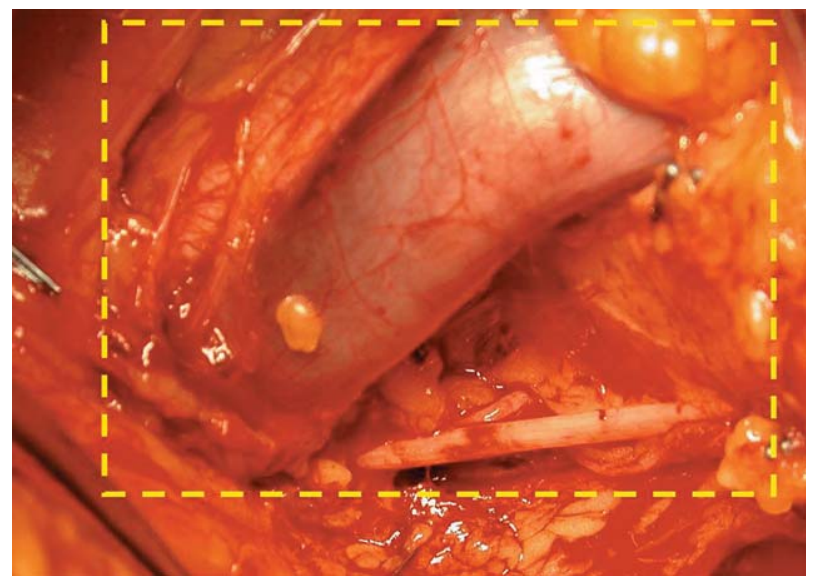

FIGURA 4. Linfadenectomia, detalle.

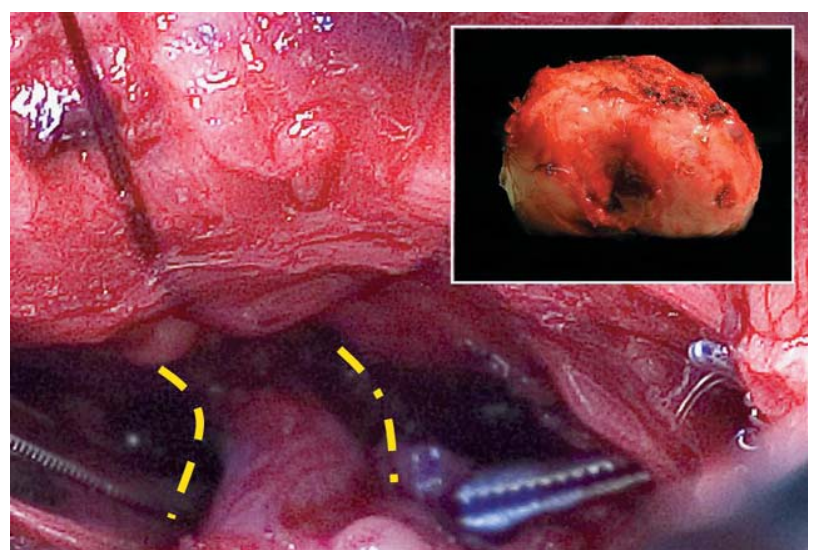

FIGURA 5. Disección de uretra. En recuadro detalle del ápex.

Con técnica combinada anterógrada y retrógrada se liberó el complejo seminal y deferencial. En todos los casos se realizó plastia de cuello vesical con 5 puntos de sutura 4/0 monofilamento. La anastomosis uretra-vesical se conformó con 5 puntos de sutura 4/0 dejando sonda vesical y comprobando estanqueidad de sutura con $150 \mathrm{cc}$. Se dejó sonda vesical durante 14-15 días. La preservación de los nervios erectores no se realizó de forma sistemática, siendo ésta indicada en casos de poca carga tumoral con menos de $30 \%$ de cilindros positivos y Gleason bajo (Fig. 7). En el último año acoplamos a la cirugía la magnificación de gafas lupa de 3x para facilitar la disección en particular de ápex y el paquete neurovascular.

Todos los pacientes siguieron en el postoperatorio el protocolo de cuidados programados establecido en el Servicio desde 1997.

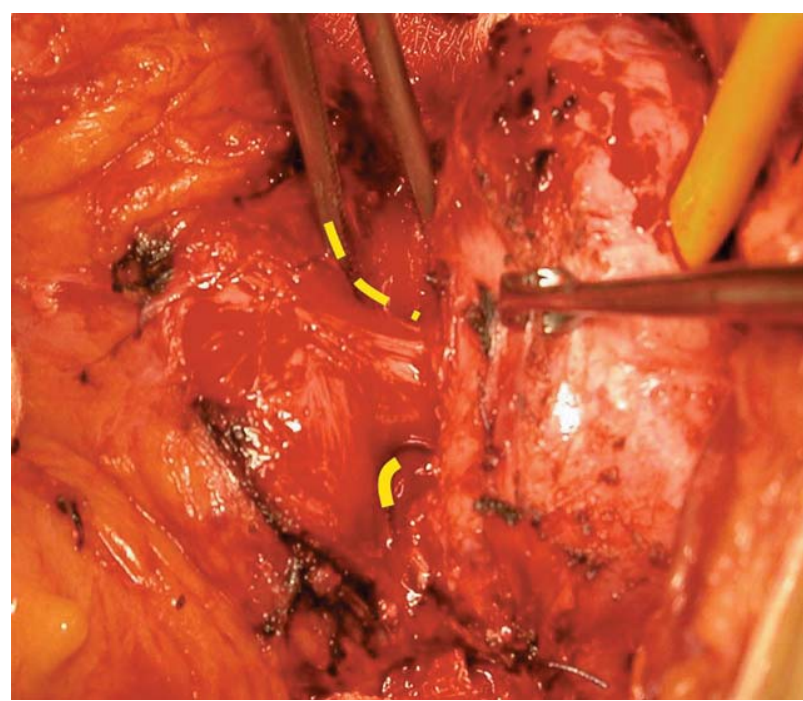

FIGURA 6. Cuello vesical preservado.

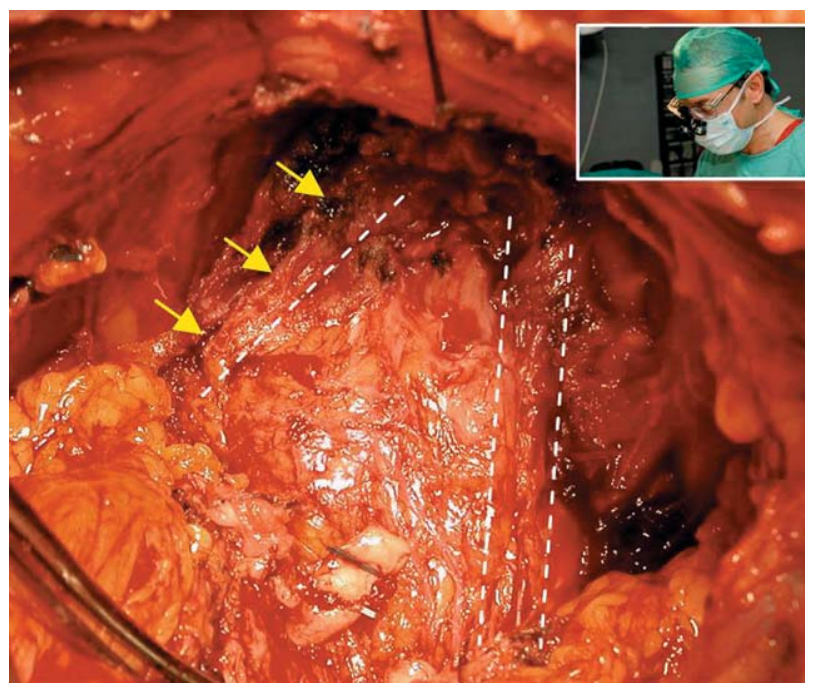

FIGURA 7. Detalle de bandeletas neuro-vasculares conservadas. Recuadro gafas lupa 3X.

\section{RESULTADOS}

La anatomía patológica correspondió (Tabla 1) en 2 casos $1,8 \%$ pT0, $42(38,2 \%)$ pT2a, 44 $40 \%$ pT2b, $16(14,5 \%)$ pT3a, $5(4,5 \%)$ pT3b y 1 $(0,9 \%)$ pT4a. El número de márgenes positivos fue de $21(19 \%)$ de los que $10(11,4 \%)$ eran en pT2 y $11(50 \%)$ en $\geq$ pT3. El Gleason postoperatorio en $39(35,5 \%)$ fue $\leq 6,49(44,5 \%) 7$, y 20 $(18,7 \%) \geq 8$.

El tiempo quirúrgico osciló entre 95 y 240 minutos, y el sangrando medio entre 850 (2002.700). Las complicaciones postoperatorias se presentaron en 9 casos (8\%) (Tabla 2). En 2 
casos fue la linforragia $(1,8 \%), 1(0,9 \%)$ linfocele, que precisó punción evacuadora. La diarrea prolongada en relación con uso antibiótico en 2 $(1,8 \%)$, en $2(1,8 \%)$ infección de orina, $1(0,9 \%)$ hemorragia postoperatoria que se resolvió con tratamiento expectante, y $1(0,9 \%)$ íleo prolongado y en otro paciente $(0,9 \%)$, tromboflebitis. No tuvimos que realizar ninguna reintervención o ampliación de la incisión. En un caso afecto por linfocele reingresó y precisó punción evacuadora. Ningún paciente fue exitus en el postoperatorio inmediato.

La estancia media fue de 4,5 días con una mediana de 4 (Tabla 3), siendo dados de alta 97 $(88,8 \%)$ en los 5 primeros días de postoperatorio. La continencia a los 6 y 12 meses se consideró completa en aquellos que no referían ninguna pérdida en el $74,2 \%$ y $80,3 \%$ respectivamente, y pérdidas mínimas con uso en ocasiones de una compresa 14,6 y $12 \%$ respectivamente; pérdidas moderadas o importantes 2 o más compresas al días $7,9 \%$ y $5,2 \%$ e incontinencia completa 3,4 y $2,4 \%$.

Tabla 2. Complicaciones perioperatorias

\begin{tabular}{lcc}
\hline Complicación & $\mathbf{N o}$ & $\mathbf{\%}$ \\
\hline Linfocele & 1 & 0,9 \\
Linforragia & 2 & 1,8 \\
Diarrea & 2 & 1,8 \\
Infección herida & 2 & 1,8 \\
Hemorragia & 1 & 0,9 \\
Ileo paralítico & 1 & 0,9 \\
Trombosis & 1 & 0,9 \\
Total & $\mathbf{9}$ & $\mathbf{8 \%}$ \\
\hline Reintervenciones & 0 & 0 \\
Reingresos & 1 & 0,9 \\
Mortalidad & 0 & 0 \\
Sin complicaciones & 101 & $90 \%$
\end{tabular}

Tabla 3. Estancia postoperatoria

\begin{tabular}{llcc}
\hline $\begin{array}{l}\text { Estancias } \\
\text { Media: }\end{array}$ & $4.5(2-19)$ & $\mathbf{N}$ & \% \\
Mediana: & 4 & & \\
\hline 2 dias & 2 & 1,8 \\
3 días & 25 & 23 \\
4 días & 49 & 45 \\
5 días & 21 & 19,1 \\
6 días & 4 & 3,6 \\
$\geq 7$ días & 9 & 8,1 \\
\hline
\end{tabular}

97 pacientes (88\%) alta en los 5 primeros días de postoperatorio.
De los 66 pacientes que previamente mantenían erecciones, $26(39,6 \%)$ mantienen erecciones en la actualidad. Con un seguimiento medio de 33 meses, a los dos años un $84,5 \%$ de los pacientes están libres de progresión y un paciente fue exitus por progresión del tumor. En un caso $(0,9 \%)$ desarrolló esclerosis de cuello que se resolvió con una cervicotomía endoscópica. y $1(0,9 \%)$ con incontinencia severa se colocó prótesis de incontinencia AMS 800.

\section{DISCUSIÓN}

El cáncer de próstata es un problema de primer orden en el mundo occidental. Dada la elevada prevalencia del mismo con cifras de nuevos diagnósticos por año superiores en España a los 13.500 y más de 5.600 defunciones en el años $2003^{2}$. Un porcentaje superior al $90 \%$ de los enfermos que se detectan son no metastáticos y por lo tanto susceptibles de tratamiento curativo $^{1}$.

La PRR es hoy por hoy el método más ampliamente utilizado para el manejo de los tumores localizados, casi la mitad de los pacientes en esta situación optan por la cirugía ${ }^{3}$. La eficacia de la $\mathrm{PR}$. en términos de supervivencia cáncer específica, ha sido puesta de manifiesto con el estudio escandinavo de reciente publicación ${ }^{6}$.

El papel predominante de la PRR. en las últimas décadas está abalado por un mejor conocimiento de la anatomía de la pelvis y en particular los estudios de Walsh ${ }^{7}$ junto con los de otros autores nos han ayudado a una mejor definición de las estructuras neuro-vasculares y complejo funcional de la continencia que han hecho posible una mejora de los resultados al facilitar la comprensión de la anatomía en su conjunto de la pelvis $^{8}$.

Todo esto hace que las cifras de mortalidad postoperatoria sean en general menores del 0,5\% oscilando entre $0-1,2 \% 9,10,11,12,13,14$. Las cifras de morbilidad postoperatoria oscilen entre el 4,9$50 \%$ constatándose para los resultados un claro efecto centro en relación a volumen de pacientes tratados, técnica utilizada y cuidados postoperatorios y volumen pacientes-cirujano ${ }^{15}$.

Si bien la PRR sigue siendo el método estándar para el tratamiento del cáncer de próstata, éste se ejecuta con incisiones infra o supraumbi- 
licales de $12-18 \mathrm{~cm}$. En el intento de minimizar la morbilidad, facilitar la recuperación del paciente con un adecuado compromiso de resultados oncológicos y funcionales se desarrollaron técnicas mínimamente invasivas como la Minilap. El concepto de Minilap basado en la idea de incisiones pequeñas de $7-8 \mathrm{~cm}$ infraumbilicales se inicia en los años 90, Steiner y Marshall ${ }^{16}$ fueron los precursores de su desarrollo. Inicialmente la técnica se desarrolla para linfadenectomías de estadiaje $^{17,18,19}$ pero los buenos resultados en términos de estancia, tiempo operatorio y complicaciones facilitan su desarrollo hacia la propia cirugía radical.

El principio básico de la misma se apoya sobre la idea de a través de una mínima incisión, conseguir un campo operatorio adecuado que nos permita acceder a las estructuras y conseguir los mismos resultados quirúrgicos que con incisiones mayores, evitando así una herida quirúrgica amplia y más dolorosa y facilitar la recuperación postoperatoria inmediata. Para ello, es necesario asumir la necesidad de una buena preparación del campo quirúrgico que difícilmente se consigue, sin una buena posición con flexión forzada en Trendelemburg y segundo, el uso de separadores multivalvas con diferentes accesorios autoretractores de diferentes tamaños que permiten adaptar el campo del paciente independientemente de su biotipo.

La incisión en la línea media se debe iniciar justo por debajo de la sinfisis del pubis para facilitar una buena separación a dicho nivel y prolongarla 7-8 cm, detalles comentados previamente en la descripción de la técnica. Es importante una vez llegados a incidir la aponeurosis de los rectos ampliar 1-2 cm la apertura de la misma aponeurosis en sus extremos, pues esta maniobra facilita el desplazamiento del resto de las estructuras. La segunda cuestión a tener en consideración tal y como comentamos en la descripción técnica, así como el propio Marshall ${ }^{5}$ es la colocación adecuada de las valvas. El objetivo de estas maniobras es fundamental para conseguir un espacio semicircular que facilite un acceso suficiente y cómodo a todas las estructuras. Existen en el mercado diferentes modelos de separadores y aditamentos específicos en tamaños y longitudes para utilizar según las circuns- tancias de cada caso. En nuestro centro, el separador se desarrollo en el mismo Hospital y disponemos de múltiples valvas que nos permiten adecuar el campo quirúrgico en función de las características de cada paciente. Todo este proceso se puede mejorar con gafas lupa que nos magnifican la visión de puntos críticos de la disección y así realizar una técnica más meticulosa en la preservación de las bandeletas neuro-vasculares, uretra prostática o cuello vesical, tal y como venimos haciendo en los últimos tiempos en nuestra serie.

Los resultados publicados con esta técnica demuestran desde el punto de vista de la morbilidad las bondades de la misma. En la serie de 552 pacientes, ninguno fue exitus en el postoperatorio. El 85\% de los pacientes presentaban continencia satisfactoria y el $88 \%$ potencia sexual mantenida. Desde el punto de vista oncológico con un mínimo de 6 meses de seguimiento, un $85 \%$ de pacientes no presentaban progresión bioquímica y un $23 \%$ tenían márgenes positivos ${ }^{5}$. La reciente revisión del mismo grupo con 200 nuevos casos entre 1999 y 2001 tiene sólo 3 (1,5\%) complicaciones postoperatorias, 3 dias de estancia media, un 10\% de márgenes positivos con un 96\% de enfermos libres de progresión y 20,5 meses de seguimiento ${ }^{20}$.

$\mathrm{Si}$ bien estos resultados son de excelencia con un volumen importante y manejados por un solo cirujano, puede obligarnos a pensar que no sean reproducibles por otras series y en diferentes entornos. Los datos obtenidos por nuestra casuística son similares a los publicados en estas series $^{5,20}$, obteniendo una cifra de complicaciones perioperatorias del 8\%, no requiriendo reintervenciones y sólo un 1 (0,9\%) de reingresos, con más de $90 \%$ de pacientes que no presentaron ninguna complicación. La mediana de estancia de 4 días es otro dato favorable a la técnica, si bien se apoya en un modelo de cuidados programados implantados en el servicio desde 1997 y que facilita la recuperación postoperatoria de los pacientes $^{21}$. La morbilidad sobre la continencia considerando globalmente no pérdidas o pérdidas esporádicas es del 92,3\% al año y la potencia sexual está preservada en el 40\% de los pacientes sexualmente activos. Desde el punto de vista oncológico un 85\% no presentaban datos de progresión. Datos similares están publicados por 
otros autores con cifras de continencia del $88 \%$ y libres de recidiva bioquímica $84 \%$, potencia sexual conservada en un $46 \%$, en el caso de Tsui ${ }^{22} \sin$ diferencias significativas en resultados comparativamente con cirugía retropúbica clásica ${ }^{23}$.

Recientemente se incide en el hecho de la menor incidencia de hernias inguinales en pacientes sometidos a PR. o Minilap, no existiendo diferencias significativas en cuanto a tiempo quirúrgico, sí en pérdidas sanguíneas $1.200 \mathrm{ml}$ sobre $1.600 \mathrm{ml}$ en los que se realizó Minilap sobre PR. clásica $(\mathrm{p}<0,01)$. La incidencia de hernias inguinales en el grupo de PR. de 17,1\% frente a un 2,9\% ( $>0,04$ ), relacionando este hecho con un menor daño producido en la vaina de los rectos al ser una incisión menor que no alteraría el conjunto de estructuras del anillo inguinal interno ${ }^{24}$.

Una evolución de la técnica de Minilap, es la realización de sutura de anastomosis a cuello uretral con endo-suturas utilizadas en cirugía laparoscópica o robótica que facilitan el acceso a la anastomosis con un tiempo de realización de aproximadamente 15 o 20 minutos, permitiendo una sutura continua y una retirada del catéter ureteral en 6 o 7 días. Esta casuística aunque corta, aporta cifras de continencia satisfactoria en los 21 pacientes estudiados ${ }^{25}$.
Otro punto a destacar es la ejecución de la técnica en pacientes con sobrepeso o índice de masa corporal superior al 30\%. También en estos casos se puede realizar dicha cirugía. En nuestra experiencia esto es factible, si bien las dificultades son mayores y precisan de una mayor adaptación del campo quirúrgico como comentamos en el desarrollo de la técnica. En cualquier caso la cirugia en pacientes con sobrepeso, tal y como se publica en la serie de Freedland sobre 7.000 pacientes tratados por 7 cirujanos, es más dificultosa y los resultados quirúrgicos peores con un mayor riesgo de afectación de la cápsula quirúrgica, incrementándose este riesgo de un $30 \%$ a un $57 \%$ según el sobrepeso sea moderado u obeso severo ${ }^{26}$.

Los resultados en general de estas técnicas son superponibles a los de las series de PRR. publicadas. Así las cifras de mortalidad perioperatoria de $0 \%$ en oscilando en las de retropúbica (1\%-0,2\%). En la serie de Augustin ${ }^{27}$ con 1.243 pacientes, un $20 \%$ presentaron complicaciones perioperatorias frente a un $1,5 \%$ y $8 \%$ en la serie de Marshall y nuestra. Un 2,9 y 0,3\% presentaron lesiones de recto en las retropúbicas, no constatándose ninguna en nuestra experiencia. El porcentaje de reintervenciones varía entre el 9\% y $0,5 \%$ en la PRR. no teniendo reintervenciones en nuestra serie ${ }^{9-14,28}$ (Tabla 4).

Tabla 4. Complicaciones perioperatorias en series de PR retropubica

\begin{tabular}{|c|c|c|c|c|c|c|c|c|c|c|}
\hline $\begin{array}{l}\text { Complicaciones } \\
\text { № (\%) }\end{array}$ & $\begin{array}{c}\text { Augustin } \\
\mathrm{N}=1243 \\
\mathrm{~N}(\%)\end{array}$ & $\begin{array}{c}\text { Andriole } \\
\mathrm{N}=1324 \\
\mathrm{~N}(\%)\end{array}$ & $\begin{array}{c}\text { Lerner } \\
N=1000 \\
N(\%)\end{array}$ & $\begin{array}{c}\text { Lepor } \\
\mathrm{N}=1000 \\
\mathrm{~N}(\%)\end{array}$ & $\begin{array}{c}\text { Hautmann } \\
\mathrm{N}=418 \\
\mathrm{~N}(\%)\end{array}$ & $\begin{array}{c}\text { Kundu } \\
\mathrm{N}=3477 \\
\mathrm{~N}(\%)\end{array}$ & $\begin{array}{c}\text { Dilliogluglil } \\
\mathrm{N}=472 \\
\mathrm{~N}(\%)\end{array}$ & $\begin{array}{c}\text { Maffezzini } \\
\mathrm{N}=300 \\
\mathrm{~N}(\%)\end{array}$ & $\begin{array}{c}\text { Rioja } \\
\mathrm{N}=216 \\
\mathrm{~N}(\%)\end{array}$ & $\begin{array}{c}\text { C.H.U. Juan } \\
\text { Canalejo } \\
\text { N=110 } \\
\text { N (\%) }\end{array}$ \\
\hline Mortalidad & 0 & $3(0,2)$ & $0(0,0)$ & $1(0,1)$ & $5(1,2)$ & 0 & $2(0,4)$ & 0 & $1(0,49)$ & 0 \\
\hline Lesiones recto & $3(0,2)$ & $3(0,2)$ & $6(0,6)$ & $5(0,5)$ & $11(2,9)$ & - & $3(0,6)$ & $1(0,3)$ & $1(0,49)$ & 0 \\
\hline $\begin{array}{l}\text { Hemorragia o } \\
\text { Hematoma }\end{array}$ & $10(0,8)$ & - & $0(0,0)$ & $3(0,3)$ & - & 1,3 & - & $2(0,7)$ & $2(1)$ & $1(0.9)$ \\
\hline Lesiones uréter & $4(0,3)$ & - & - & $1(0,1)$ & $1(0,2)$ & - & $1(0,2)$ & $1(0,3)$ & - & 0 \\
\hline Ileo paralítico & $4(0,3)$ & - & - & $2(0,2)$ & - & - & $15(3,2)$ & - & $1(0,49)$ & $1(0.9)$ \\
\hline Infarto de miocardio & $5(0,4)$ & $9(0,7)$ & $7(0,7)$ & $2(0,2)$ & $3(0,7)$ & $3(0,1)$ & $2(0,4)$ & - & - & 0 \\
\hline Embolismo pulmonar & $2(0,2)$ & $22(1,7)$ & $6(0,6)$ & $2(0,2)$ & $6(1,4)$ & - & $5(1,0)$ & $1(0,3)$ & - & 0 \\
\hline Tromboflebitis & $12(1)$ & $8(0,6)$ & $14(1,4)$ & $1(0,1)$ & $7(1,7)$ & $45(1,3)$ & $6(1,3)$ & - & $1(0,49)$ & $1(0.9)$ \\
\hline Sepsis o absceso & $3(0,2)$ & - & $2(0,5)$ & - & - & - & $3(0,6)$ & - & $3(1,47)$ & 0 \\
\hline $\begin{array}{l}\text { Infección herida o } \\
\text { dehiscencia }\end{array}$ & $25(2)$ & $17(1,3)$ & $9(0,9)$ & $8(0,8)$ & $11(2,6)$ & - & $14(2,9)$ & $3(1)$ & $2(0.98)$ & $2(1.8)$ \\
\hline Linfocele & $29(2.3)$ & - & - & $1(0,1)$ & $28(6,7)$ & $7(0,01)$ & $10(2,1)$ & $3(1)$ & $3(1,47)$ & $1(0,9)$ \\
\hline Linforragia & $16(1,3)$ & $8(0.6)$ & - & - & - & - & $3(0,6)$ & - & - & $2(1,8)$ \\
\hline $\begin{array}{l}\text { Retirada prematura } \\
\text { de catéter }\end{array}$ & $1(0.1)$ & $4(0,3)$ & - & - & - & - & $7(1,5)$ & - & - & 0 \\
\hline Reintervenciones & $32(2,6)$ & - & - & $10(1)$ & $46(9)$ & - & $3(1,6)$ & $5(1,7)$ & $1(0,49)$ & 0 \\
\hline
\end{tabular}


Los datos funcionales respecto a la continencia son similares a los publicados en PRR. que varían entre el 93\% en cifras recientes de la serie de Kundu ${ }^{29}$ con 3.477 paciente evaluados a datos de $82 \%^{14}$ comunicados por otros grupos, siendo estos resultados equiparables a los presentados por las series de Minilap con cifras de 97,3\% a $88 \%$, siendo las nuestras propias al año de un $92,3 \%$

En lo relacionado a los datos oncológicos de las series de Minilap, si bien tienen seguimientos más cortos, los datos de progresión presentan valores asumibles en los diferentes intervalos de seguimiento, 97\% libres de progresión en la serie de Slabughh ${ }^{20}$ con 20,5 meses de media de seguimiento, siendo nuestra experiencia de $84,5 \%$ a los dos años, con un seguimiento medio de 33 meses. Los datos de márgenes positivos publicados en las cirugias retropúbicas oscilan entre el $10 \%-49 \% \%^{30,31}$, siendo en la nuestra un $19 \%$ con un $11,4 \%$ en pacientes pT2.

Las cifras de pacientes que pueden presentar vida sexual activa tras la $\mathrm{PR}$ retropúbica varían según autores entre el 10\%-86\% ${ }^{31,32}$ en relación a centros con mayor o menor afinidad a la preservación de los plexos neuro-vasculares. Los datos en las series de Minilap oscilan entre el $40 \%-76 \%{ }^{20}$. En nuestra experiencia que ha seguido criterios oncológicos inicialmente, y por tanto no en todos los casos se intentó una preservación de las estructuras, un $40 \%$ de los pacientes con actividad sexual previa pueden al año mantenerla, la aplicación de la magnificación con gafas lupa puede contribuir a la mejora de los resultados pero en cualquier caso la Minilap no tiene por qué ser una dificultad especial añadida para esta técnica.

La aparición de la cirugía laparoscópica ha supuesto un revulsivo en el contexto de la comunidad urológica en los últimos años, con la proliferación de grupos dedicados a su aplicación. Comparando diferentes series de excelencia con volúmenes amplios de cirugías, el número de complicaciones postoperatorias, estancias o reintervenciones no presentan mejores resultados que los aportados por las series de Minilap, destacando los porcentajes de reintervenciones en las laparoscópicas de 3,7\% a $1,7 \%$ y siendo los tiempos quirúrgicos consumidos mayores, no incluyendo en muchos de los casos linfadenectomías de estadiaje ${ }^{33-35}$ (Tabla 5). Si bien es ésta una cirugía en desarrollo y conocida su curva de

Tabla 5. Comparación entre prostatectomía radical Minilap y Laparoscópica: Resultados quirúrgicos.

\begin{tabular}{|c|c|c|c|c|c|}
\hline & & & & & \\
\hline & Slabaugh & $\begin{array}{l}\text { C.H.U. JUAN } \\
\text { CANALEJO }\end{array}$ & Guillonneau & $\begin{array}{l}\text { Rassweiler } 1 \\
\text { Ultima serie }\end{array}$ & Rozet \\
\hline № pacientes & 209 & 110 & 567 & 219 & 600 \\
\hline Tiempo intervención (min)* & 166 & 160 & 244 & 218 & 173 \\
\hline Pérdidas sanguíneas (cc)* & 995 & 850 & 380 & 800 & 380 \\
\hline \% Transfusión & 4 & - & $4.9(28)$ & 9.6 & - \\
\hline \% Conversión a cirugía & 0 & - & 2,4 & 0.5 & 0.1 \\
\hline \% Complicaciones (nº) & & & & & \\
\hline Retención & 0 & 0 & $4.6(26)$ & - & $3(20)$ \\
\hline Hematoma & $0.5(1)$ & $1(1.2)$ & & (3) & 2 \\
\hline Lesión ureteral & 0 & 0 & $0.5(3)$ & - & - \\
\hline Lesión intestinal & 0 & 0 & $1.4(11)$ & $(0)$ & $1(4)$ \\
\hline Infección herida & 0 & 2.7 & $0.7(4)$ & - & - \\
\hline Ileo & $1(2)$ & $1(1.2)$ & $1(6)$ & - & 2 \\
\hline Fístula anastómica & 0 & 0 & - & - & $5(30)$ \\
\hline Trombosis venosa & $0.8(1)$ & $2(1,8)$ & - & - & - \\
\hline Embolismo & 0 & 0 & - & (1) & 1 \\
\hline Estancia postoperatoria & 3.0 & 4 & 6,2 & 11 & 6.3 \\
\hline$\%$ Reintervenciones ( $\mathrm{n}^{\circ}$ ) & 0 & 0 & $3,7(21)$ & $1,8(4)$ & $1,7(10)$ \\
\hline
\end{tabular}

\footnotetext{
* Medio
} 
aprendizaje prolongada, costes elevados y dificultad de desarrollo, volúmenes importantes de pacientes para su adecuado aprendizaje, no siempre es posible en un porcentaje elevado de centros con menos volúmenes de patología. Es posible que sea más fácil adaptar el conocimiento de la cirugía retropúbica hacia técnicas como la Minilap para cirujanos adaptados a cirugía retropúbica.

Una reflexión final que nos ha de guiar en el desarrollo de cualquier técnica es el poder ofrecer y optimizar nuestros resultados a los pacientes. En este sentido, y a pesar de la mejora en todos nuestros conocimientos y resultados favorables de nuestras series, los resultados óptimos entendido por tal los pacientes que son totalmente continentes, no tienen datos de progresión de enfermedad y que mantenían vida sexual activa la siguen manteniendo sólo se consigue en un 30\% de pacientes al año y un 53\% a los cuatro años de la cirugía, según recientes estudios referidos a centros terciarios ${ }^{36}$. Por tanto debemos seguir mejorando nuestras técnicas quirúrgicas y métodos de selección de los tratamientos a ofertar.

\section{CONCLUSIONES}

La Minilap es una técnica válida para la realización de la PR. retropúbica. El conocimiento previo y la familiaridad de la cirugía retropúbica con incisiones mayores facilitan su aprendizaje. Es fundamental disponer y adaptarse al uso de separadores específicos que facilitan la preparación de un campo más limitado, que no insuficiente para la cirugia. El hecho de menores incisiones puede ayudar a una más rápida recuperación del enfermo en el postoperatorio con estancias más cortas y menor morbilidad perioperatoria.

La mejora de la calidad del trabajo no siempre deben de ir asociados con técnicas más costosas, difíciles de reproducir en centros con volúmenes de pacientes más reducidos y cuyos resultados globales no están estandarizados.

La Minilap es una técnica fácilmente reproducible con resultados funcionales y oncológicos similares a los del estándar de la PRR.

\section{REFERENCIAS}

1. Jemal A, Siegel R, Ward E, Murray T, Xu J, Smigal C, Thun MJ. Cancer Statistics, 2006. CA Cancer J Clin. 2006;56(2): 106-130.

2. Centro Nacional de Epidemiología. La situación del cáncer en España. Madrid: Ministerio de Sanidad y Consumo, 2005.
3. Meng MV, Elkin EP, Harlan SR et al. Predictors of treatment alter inicial surveillance in men with prostate cancer: Results from CapSURE. J Urol. 2003 Dec;170(6 Pt 1): 2279-2283.

4. Janet L, Stanford JL, Feg Z, Hamilton AS, Gilliland FD, Stephenson RA, Eley JW, et al. Urinary and Sexual Function After Radical Prostatectomy for Clinically Localized Prostate Cancer. JAMA. 2000 Jan;283(3):354-360.

5. Marshall FF, Chan D, Partin AW, Gurganus R, Hortopan SC. Minilaparotomy radical. J Urol. 1998 Dec;160(6 Pt 2): 2440-2445.

6. Bill-Axelson A, Holmberg L, Ruutu M, Haggman M, Andersson SO, Bratell S, Spangberg A, Busch C, Nordling $\mathrm{S}$, Garmo H, Palmgren J, Adami HO, Norlen BJ, Johansson JE; Scandinavian Prostate Cancer Group Study No. 4. Radical prostatectomy versus watchful waiting in early prostate cancer. N Engl J Med. 2005 May 12; 352(19):1977-1984.

7. Walsh PC, Donker PJ. Impotence following radical prostatectomya: insight into etiology and prevention. J Urol. 1982 Sep;128(3):492-497.

8. Castiñeiras J, López A, Ronco A, Martín A, Varo C, González D, Palomo MJ, Rodríguez-Rubio F. La prostatectomía radical retropúbica. II-La neuroanatomía del plexo pélvico, la impotencia y la incontinencia urinaria. Actas Urol Esp. 1994;18(supl):444-451.

9. Andriole GL, Smith DS, Rao G, Goodnough L, Catalona WJ. Early complications of contemporary anatomical radical retropubic prostatectomy. Urol. 1994 Nov;152(5 Pt 2): 1858-1860

10. Lerner SE, Blute ML, Lieber MM, Zincke H. Morbidity of contemporary radical retropubic prostatectomy for localized prostate cancer. Oncology. (Williston Park). 1995 May; 9(5):379-382.

11. Lepor H, Nieder AM, Ferrandino MN. Intraoperative and postoperative complications of radical retropubic prostatectomy in a consecutive series of 1,000 cases. J Urol. 2001; 166(5):1729-1733.

12. Hautmann RE, Sauter TW, Wnderoth UK. Radical retropubic prostatectomy: morbidity and urinary continence in 418 consecutive cases. Urology. 1994;43(2 suppl):47-51.

13. Dillioglugil O, Leibman BD, Leibman NS, Kattan MW, Rosas AL, Scardino PT. Risk factors for complications and morbidity alter radical retropubic prostatectomy. J Urol. 1997;157(5):1760-1767.

14. Rioja LA, Liedana JM, Roncales A, Gil MJ, Villanueva A, Rodriguez L, Gil P. Análisis de una serie de prostatectomías radicales. Actas Urol Esp. 1997;21(9):809-816.

15. Bianco FJ, Riedel ER, Begg CB, Kattan MW, Scardino PT. Variations among high volume surgeons in the rate of complications after radical prostatectomy: further evidence that technique matters. J Urol. 2005;173(6):2099-2103.

16. Steiner MS, Marshall FF. Mini-laparotomy staging pelvic lymphadenectomy (minilap): alternative to standard laparoscopic pelvic lymphadenectomy. Urology. 1993;41(3): 20012006 .

17. Idom CB Jr, Steiner MS. Minilaparotomy staging pelvic lymphadenectomy follow-up: a safe alternative to standard and laparoscopic pelvic lymphadenectomy. World J Urol. 1998; 16:396-399.

18. Lezin MS, Cherrie R, Cattolica EV. Comparison of laparoscopic and minilaparotomy pelvic lymphadenectomy for prostate cancer staging in a community practice. Urology. 1997;49:60-64. 
19. Perrotti M, Gentle DL, Barada JH, Wilbur HJ, Kaufman RP. Mini-laparotomy pelvic lymph node dissection minimizes morbidity, hospitalisation, and cost of pelvic lymph node dissection. J Urol. 1996;196:986-988.

20. Slabaugh TK, Marshall FF. A comparison of minimally invasive open and laparoscopic radical retropubic prostatectomy. J Urol. 2004;172(6 Pt 2):2545-2548.

21. Gómez Veiga F, Castelo LA, Ruibal M, Rivera J, Chantada V, Martín MG. Reduction in length of stay with clinical care pathways for radical retropubic prostatectomy. Eur Urol. 2000;37(S2):99.

22. Tsui KH, Sun GH, Huang ST, Wang TM, Lee SH, Chang PL. Minilaparotomy radical retropubic prostatectomy for prostate cancer. Arch Androl. 2004;50:23-25.

23. Maffezzini M, Graziotti P, Seveso M, Giusti G, Taberna G, Benetti A. A prospective comparison of standard and minilap radical retropubic prostatectomy. Eur Urol. 2003;(S2): 63.

24. Nomura T, Mimata H, Kitamura H, Fujikura Y, Akita Y, Yamasaki M, et al. Lower incidente of inguinal hernia: minilaparotomy radical retropubic prostatectomy compared with convencional technique. Urol Int. 2005;74(1): 32-37.

25. Miki T, Okihara K, Ukimura O, Usijima S, Yoneda K, Mizutani $\mathrm{Y}$ et al. Running suture for vesicourethral anastomosis in minilaparotomy radical retropubic prostatectomy. Urology. 2006;47:410-412.

26. Freedland SJ, Grubb KA, Yiu SK, Nielsen ME, Mangold LA, Isaacs WB, Epstein JI, Partin AW. Obesity and capsular incision at the time of open retropubic radical prostatectomy. J Urol. 2005 Nov;174(5):1798-1801; discussion 1801.

27. Augustin H, Hammerer P, Graefen M, Palisaar J, Noldus J, Fernandez $\mathrm{S}$ et al. Intraoperative and perioperative morbidity of contemporary radical retropubic prostatectomy in a consecutive series of 1243 patients: results of a single center between 1999 and 2002. Eur Urol. 2003 Feb;43(2): 113-118.

28. Maffezzini M, Seveso M, Taverna G, Giusti G, Benetti A, Graziotti P. Evaluation of complications and results in a contemporary series of 300 consecutive radical retropubic prostatectomies with the anatomic approach at a single institution. Urology. 2003 May;61(5):982-986.
29. Kundu SD, Roehl KA, Eggener SE, Antenor JA, Han M, Catalona WJ. Potency, continence and complications in 3,477 consecutive radical retropubic prostatectomies. J Urol. 2004 Dec;172(6 Pt 1):2227-2231.

30. Eastham JA, Kattan MW, Riedel E, Begg CB, Wheeler TM, Gerigk C, et al. Variations among individual surgeons in the rate of positive surgical margins in radical prostatectomy specimens. J Urol. 2003 Dec;170(6 Pt 1):2292-2295.

31. Aguiló Lucia F, Suárez Novo JF, Planes Morín J, Condom Mundó E. Prostatectomía radical. Revisión de nuestra serie en el periodo 1997-2003. Actas Urol Esp. 2005 Jun; 29(6):542-549.

32. Walsh PC, Marschke P, Ricker D, Burnett AL. Patientreported urinary continence and sexual function after anatomic radical prostatectomy. Urology. 2000 Jan;55(1): 5861.

33. Guillonneau B, Rozet F, Cathelineau X, Lay F, Barret E, Doublet JD, et al. Perioperative complications of laparoscopic radical prostatectomy: the Motsouris 3-year experience. J Urol. 2002 Jan;167(1):51-56.

34. Rassweiler J, Seemann O, Shulze M, Teber D, Hatzinger M, Frede T. Laparoscopic versus open radical prostatectomy: a comparative study at a single institution. J Urol. 2003 May; 169(5): 1689-1993.

35. Rozet F, Galiano M, Cathelineau X, Barret E, Cathala N, Vallancien G. Extraperitoneal laparoscopic radical prostatectomy: a prospective evaluation of 600 cases. J Urol. 2005 Sep; 174(3):908-911.

36. Saranchuk JW, Kattan MW, Elkin E, Touijer K , Scardino PT, Eastham J. Achieving optimal outcomes after radical prostatectomy. J Clin Oncol. 2005 Jun 20;23(18):41464151.

Correspondencia autor: Dr. F. Gómez Veiga Servicio de Urología.

Complexo Hospitalario Universitario Juan Canalejo

Xubias de Arriba, 84 - 15006 A Coruña

Tel.: 981178000

E-mail autor: f.veiga@telefonica.net

Informaión artículo: Original 